\title{
Dirhodium Tetracarboxylates Derived from Adamantylglycine as Chiral Catalysts for Enantioselective C-H Aminations
}

\author{
Ravisekhara P. Reddy and Huw M. L. Davies*
}

\author{
Supporting Information
}

Experimental Procedures

Spectral Data
S2-S1 1

S12-S25 


\section{General Procedure:}

${ }^{1} \mathrm{H}$ NMR spectra were run at either 400 or $500 \mathrm{MHz}$, and ${ }^{13} \mathrm{C} \mathrm{NMR}$ at either 75 or $125 \mathrm{MHz}$ with the sample solvent being $\mathrm{CDCl}_{3}$ unless otherwise noted. Mass spectral determinations were carried out in GC-MS (EI), LC-MS (ESI) or by Instrument Center, Department of Chemistry, University at Buffalo. IR spectra were obtained using a Perkin Elmer 1760X FT-IR. Optical rotations were measured using a Jasco DIP-370 digital polarimeter. Elemental analyses were performed by Atlantic Microlabs Inc., Norcross GA. Enantiomeric excess was determined by HPLC (UV detection at $254 \mathrm{~nm}$ ). Analytical TLC was performed on $0.25 \mathrm{~mm}$ E. Merck silica gel (60F-254) plates using UV light.

Glassware was dried in oven overnight then flame or heat-gun dried prior to use. Reactions were conducted under argon atmosphere. Column chromatography was carried out on Merck silica gel 60 (230-400 mesh). Solvents THF, $\mathrm{Et}_{2} \mathrm{O}, \mathrm{CH}_{3} \mathrm{CN}, \mathrm{CH}_{2} \mathrm{Cl}_{2}$ and toluene were dried by solvent purifier.

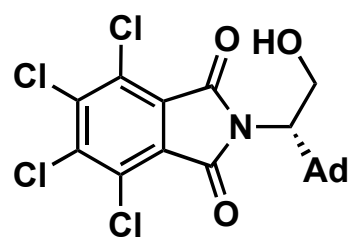

\section{2-((S)-1-Adamantyl-2-hydroxyethyl)4,5,6,7-tetrachloroisoindoline-1,3-dione:}

Tetrachlorophthalic anhydride $(0.76 \mathrm{~g}, 5.12 \mathrm{mmol}, 1.0$ equiv. $)$ was added to a solution of (S)-2-amino-2-adamantylethanol (1.0 g, $5.12 \mathrm{mmol}, 1.0$ equiv.) in DMF (6 mL) and heated at $140{ }^{\circ} \mathrm{C}$ for $12 \mathrm{~h}$. After cooling, the reaction mixture was poured into water, and the product precipitated out as a white solid. The crystals were filtered and vacuum dried to give the product $(1.9 \mathrm{~g}, 80 \%$ yield $)$ as a white sticky solid. $\mathrm{R}_{\mathrm{f}}=0.35(3: 1$ 
hexane/EtOAc); $[\alpha]_{\mathrm{D}}^{25}-4.5^{\circ}\left(c\right.$ 0.22, $\left.\mathrm{CHCl}_{3}\right)$; IR (neat) $3273,2903,2849,1641,1543$, 1401, 1343, 1266, $1132 \mathrm{~cm}^{-1} ;{ }^{1} \mathrm{H}$ NMR $\left(\mathrm{CDCl}_{3}, 500 \mathrm{MHz}\right) \delta 4.53(\mathrm{t}, J=13.5 \mathrm{~Hz}, 1 \mathrm{H})$, $4.04(\mathrm{dd}, J=5.5,13.5 \mathrm{~Hz}, 1 \mathrm{H}), 3.98(\mathrm{dd}, J=5.5,13.5 \mathrm{~Hz}, 1 \mathrm{H}), 1.98(\mathrm{bs}, 3 \mathrm{H}), 1.71-1.60$ (m, $13 \mathrm{H}) ;{ }^{13} \mathrm{C}$ NMR $\left(125 \mathrm{MHz}, \mathrm{CDCl}_{3}\right) \delta$ 165.1, 129.7, 127.4, 127.3, 64.4, 57.5, 40.1, 37.1, 36.7, 28.3; HRMS (ESI) $m / z$ Calcd for $\left[\mathrm{C}_{20} \mathrm{H}_{20} \mathrm{Cl}_{4} \mathrm{NO}_{3}\right]^{+}\left[(\mathrm{M}+\mathrm{H})^{+}\right]$: 462.0197 . Found: 462.0201.

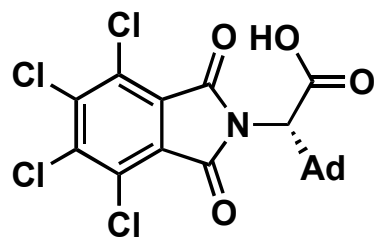

\section{(S)-Adamantan-1-yl-(4,5,6,7-tetrachloro-1,3-dioxo-1,3-dihydro-isoindol-2-yl)-acetic}

acid: $\mathrm{NaIO}_{4}(3.4 \mathrm{~g}, 16.0 \mathrm{mmol}, 4.1 \mathrm{eq})$ in water $(25 \mathrm{~mL})$ was added to a stirring solution of 2-((S)-1-adamantyl-2-hydroxyethyl) 4,5,6,7-tetrachloroisoindoline-1,3-dione (1.8 g, $3.9 \mathrm{mmol})$ in $(1: 1) \mathrm{EtOAc} / \mathrm{CH}_{3} \mathrm{CN}(34 \mathrm{~mL})$, and stirred at $23{ }^{\circ} \mathrm{C}$ for $10 \mathrm{~min} . \mathrm{RuCl}_{3} \cdot \mathrm{H}_{2} \mathrm{O}$ $(0.02 \mathrm{~g}, 2.2 \mathrm{~mol} \%)$ was then added and stirred vigorously for $12 \mathrm{~h}$. The reaction mixture was diluted with DCM and filtered through a pad of celite and charcoal. The filtrate was washed with water and brine and dried over anhydrous $\mathrm{MgSO}_{4}$, and the solvent was removed in vacuo. The resulting residue was dissolved in ether $(25 \mathrm{~mL})$ and filtered through a pad of celite and charcoal. The solvent was then concentrated to give the product $(1.26 \mathrm{~g}, 68 \%)$ as a white solid. $\mathrm{R}_{\mathrm{f}}=0.17(1: 1$ hexane/EtOAc $) ;[\alpha]_{\mathrm{D}}^{25}-14.4^{\circ}(c$ 0.15, $\left.\mathrm{CHCl}_{3}\right)$; IR (neat): 2906, 2851, 1723, 1387, 1370, $737 \mathrm{~cm}^{-1} ;{ }^{1} \mathrm{H}$ NMR $\left(\mathrm{CDCl}_{3}, 400\right.$ MHz) $\delta 4.57(\mathrm{~s}, 1 \mathrm{H}), 1.99-1.66(\mathrm{~m}, 15 \mathrm{H}) ;{ }^{13} \mathrm{C} \mathrm{NMR}\left(125 \mathrm{MHz}, \mathrm{CDCl}_{3}\right) \delta 170.9,163.5$, 140.5, 130.0, 127.2, 61.2, 39.3, 37.8, 36.5, 28.4; HRMS (EI) $\mathrm{m} / \mathrm{z}$ Calcd for $\mathrm{C}_{20} \mathrm{H}_{17} \mathrm{Cl}_{3} \mathrm{NO}_{4}{ }^{37} \mathrm{Cl}_{1}: 476.9877$. Found: 476.9862. 


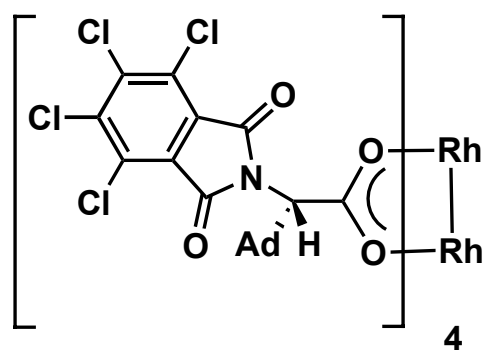

$\mathbf{R h}_{2}(\mathbf{S}-\mathrm{TCPTAD})_{4}(\mathbf{4 b})$. The following procedure is similar to that reported by Callot. ${ }^{1}$ (S)-adamantan-1-yl-(4,5,6,7-tetrachloro-1,3-dioxo-1,3-dihydro-isoindol-2yl)-acetic acid (1.2 g, $2.5 \mathrm{mmol}, 6$ equiv.) and $\mathrm{Rh}_{2}(\mathrm{OAc})_{4}(188 \mathrm{mg}, 0.42 \mathrm{mmol})$ were dissolved in dry chlorobenzene $(12 \mathrm{~mL})$ in a flask under argon, and stirred at $23{ }^{\circ} \mathrm{C}$ for $30 \mathrm{~min}$ and then the mixture was heated up to $150{ }^{\circ} \mathrm{C}$ and the acetic acid was distilled out as an azeotrope with chlorobenzene for $3 \mathrm{~h}$. Additional $25 \mathrm{~mL}$ was added and distilled during the reaction. The mixture was cooled and the solvent was removed in vacuo. The residue was subjected to flash chromatography (silica, 1.5:1 hexanes / EtOAc - 1:1 hexanes / EtOAc) to give $4 \mathbf{b}(0.55 \mathrm{~g}, 62 \%)$ as a bright green solid. $\mathrm{R}_{\mathrm{f}}=0.7\left(1: 3\right.$ hexanes:EtOAc); $[\alpha]_{\mathrm{D}}{ }^{25}$ $+82.6^{\circ}$ (c 0.19, $\left.\mathrm{CHCl}_{3}\right)$; FTIR: 2904, 2850, 1726, 1610, 1370, 1200, $740 \mathrm{~cm}^{-1} ;{ }^{1} \mathrm{H}$ NMR $\left(\mathrm{CDCl}_{3}, 400 \mathrm{MHz}\right) \delta 4.70(\mathrm{~s}, 1 \mathrm{H}), 1.99-1.67(\mathrm{~m}, 15 \mathrm{H}) ;{ }^{13} \mathrm{C} \mathrm{NMR}\left(125 \mathrm{MHz}, \mathrm{CDCl}_{3}\right) \delta$ $186.2,163.4,162.8,140.2,139.8,130.1,129.3,127.3,62.3,39.3,38.4,36.9,28.5$; HRMS (FAB) calc for $\left[\mathrm{C}_{80} \mathrm{H}_{64} \mathrm{Cl}_{16} \mathrm{~N}_{4} \mathrm{O}_{16} \mathrm{Rh}_{2}\right]^{+}\left([\mathrm{M}+\mathrm{H}]^{+}\right)$2102.7522. Found: 2102.7536.

\section{General procedure for the intermolecular $\mathbf{C}-\mathrm{H}$ amination:}

A solution of $\mathrm{PhI}(\mathrm{OAc})_{2}(1.5$ equiv. $)$ in trifluorotoluene $(10 \mathrm{~mL})$ was added to a solution of substrate (5 equiv.) $\mathrm{NsNH}_{2}$ ( 1 equiv.) $\mathrm{MgO}$ (2.3 equiv.) and the catalyst ( $\left.2 \mathrm{~mol} \%\right)$ in trifluorotoluene $(15 \mathrm{~mL})$ at $23{ }^{\circ} \mathrm{C}$ over $0.5 \mathrm{~h}$ using a syringe pump. The reaction mixture

\footnotetext{
${ }^{1}$ Callot, H. J.; Metz, F. Tetrahedron 1985, 41, 4495.
} 
was allowed to stir for $3 \mathrm{~h}$ and then filtered to remove the precipitated solids and the filtrate was concentrated. The residue was purified using flash column chromatography.

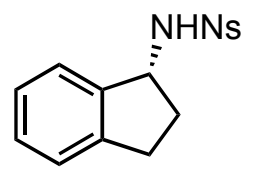

(R)- $\boldsymbol{N}$-Indan-1-yl-4-nitro-benzenesulfonamide (5). ${ }^{1} \mathrm{H} \mathrm{NMR}\left(\mathrm{CDCl}_{3}, 400 \mathrm{MHz}\right) \delta 8.38$ $(\mathrm{d}, J=8.8 \mathrm{~Hz}, 2 \mathrm{H}), 8.12(\mathrm{~d}, J=8.8 \mathrm{~Hz}, 2 \mathrm{H}), 7.15-7.26(\mathrm{~m}, 3 \mathrm{H}), 7.09(\mathrm{~d}, J=7.2 \mathrm{~Hz}, 1 \mathrm{H})$, 4.93-4.89 (m, 2H), 2.97-2.90 (m, 1H), 2.85-2.76 (m, 1H), 2.35-2.42 (m, 1H), 1.76$1.82(\mathrm{~m}, 1 \mathrm{H}) ;[\alpha]_{\mathrm{D}}^{25}+16.4^{\mathrm{o}}\left(c \quad 0.68, \mathrm{CHCl}_{3}, 94 \%\right.$ ee $)$. Lit. $[\alpha]_{\mathrm{D}}+22.7^{\circ}\left(\mathrm{c} 1.30, \mathrm{CHCl}_{3}\right){ }^{2}$ HPLC analysis: $94 \%$ ee. Chiralcel AD-H, $25.0 \% i$-PrOH, $0.8 \mathrm{~mL} / \mathrm{min}, 12.6 \mathrm{~min}$ (major), 18.3 (minor). The NMR data are consistent with the published data. ${ }^{3}$<smiles>C[C@H](N[NH3+])c1ccccc1</smiles>

(R)- $\mathrm{N}$-(1-Phenyl-ethyl)- 4-nitro-benzenesulfonamide (7a). ${ }^{1} \mathrm{H} \mathrm{NMR}\left(\mathrm{CDCl}_{3}, 500 \mathrm{MHz}\right)$ $\delta 8.40(\mathrm{~d}, J=8.5 \mathrm{~Hz}, 2 \mathrm{H}), 8.12(\mathrm{~d}, J=8.5 \mathrm{~Hz}, 2 \mathrm{H}), 7.18-7.15(\mathrm{~m}, 2 \mathrm{H}), 7.10-7.06(\mathrm{~m}$, 2H), $6.95(\mathrm{~d}, J=7.5 \mathrm{~Hz}, 1 \mathrm{H}), 4.84(\mathrm{~d}, J=8.0 \mathrm{~Hz}, 1 \mathrm{H}), 4.58-4.55(\mathrm{~m}, 1 \mathrm{H}), 2.77-2.69(\mathrm{~m}$, $3 \mathrm{H}) ;[\alpha]_{\mathrm{D}}^{25}+11.6^{\mathrm{o}}\left(c 0.73, \mathrm{CHCl}_{3}\right)$. Lit $[\alpha]_{\mathrm{D}}+6.23^{\circ}\left(\mathrm{c} 0.80, \mathrm{CHCl}_{3}\right) ;^{2} \mathrm{HPLC}$ analysis: 74 \% ee. Chiralcel OD-H, $1.0 \%$ i-PrOH, $0.7 \mathrm{~mL} / \mathrm{min}, 5.8 \mathrm{~min}$ (major), $11.1 \mathrm{~min}$ (minor). The NMR data are consistent with the published data. ${ }^{3}$

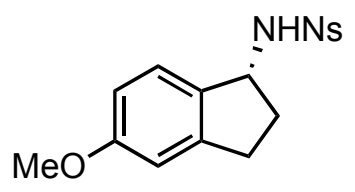

\footnotetext{
${ }^{2}$ Yamawaki, M.; Tsutsui, h.; Kitagaki, S.; Anada, M.; Hashimoto. S. Tetrahedron Lett. 2002, 42, 9561.

${ }^{3}$ Nageli, I.; Baud, C.; Bernardinelli, G.; Jacquier, Y.; Moran, M.; Muller, P. Helv. Chim. Acta 1997, 80, 1087.
} 
(R)- $N$-(5-Methoxy-indan-1-yl)-4-nitrobenzenesulfonamide (7b). white solid; $\mathrm{mp}=95$ $98{ }^{\circ} \mathrm{C} ; \mathrm{R}_{\mathrm{f}}=0.40$ (2:1 hexanes:EtOAc); $[\alpha]_{\mathrm{D}}^{25}+44.9^{\circ}$ (c 0.12, acetone); FTIR: 3286, 2946, 1607, 1530, 1493,1434, 1349, 1164, 1093, 1029, 851, 736, 643, $619 \mathrm{~cm}^{-1} ;{ }^{1} \mathrm{H}$ NMR $\left(\mathrm{D}_{2} \mathrm{O}, 500 \mathrm{MHz}\right) \delta 8.38(\mathrm{~d}, J=7.0 \mathrm{~Hz}, 2 \mathrm{H}), 8.12(\mathrm{~d}, J=7.0 \mathrm{~Hz}, 2 \mathrm{H}), 6.97(\mathrm{~d}, J=$ $8.0 \mathrm{~Hz}, 1 \mathrm{H}), 6.70(\mathrm{~m}, 2 \mathrm{H}), 4.86(\mathrm{~s}, 2 \mathrm{H}), 3.77(\mathrm{~s}, 3 \mathrm{H}), 2.89(\mathrm{~m}, 1 \mathrm{H}), 2.77(\mathrm{~m}, 1 \mathrm{H}), 2.36(\mathrm{~m}$, $1 \mathrm{H}), 1.79(\mathrm{~m}, 1 \mathrm{H}) ;{ }^{13} \mathrm{C} \mathrm{NMR}\left(125 \mathrm{MHz}, \mathrm{CDCl}_{3}\right) \delta 160.1,157.1,147.2,145.3,133.0$ 128.1, 125.1, 124.6, 103.1, 100.4, 58.8, 55.9, 35.5, 30.1; HRMS (EI) $\mathrm{m} / z$ calc for $\left[\mathrm{C}_{16} \mathrm{H}_{16} \mathrm{~N}_{2} \mathrm{O}_{5} \mathrm{~S}\right]^{+}\left[(\mathrm{M})^{+}\right]$: 348.0774. Found: 348.0779; HPLC analysis: Chiralcel AD-H, $25.0 \% \mathrm{ipa}, 0.7 \mathrm{~mL} / \mathrm{min}, 24.0 \mathrm{~min}$ (major), $26.3 \mathrm{~min}$ (minor).

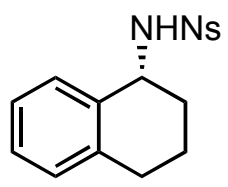

(R)-N-(1,2,3,4-Tetrahydronaphthalene-1-yl)-4-nitrobenzenesulfonamide $\quad(7 \mathrm{c}) . \quad{ }^{1} \mathrm{H}$ NMR $\left(\mathrm{CDCl}_{3}, 400 \mathrm{MHz}\right) \delta 8.40(\mathrm{~d}, J=8.8 \mathrm{~Hz}, 2 \mathrm{H}), 8.12(\mathrm{~d}, J=8.8 \mathrm{~Hz}, 2 \mathrm{H}), 7.17(\mathrm{t}, J=$ $7.2 \mathrm{~Hz}, 1 \mathrm{H}), 7.10-7.05(\mathrm{~m}, 2 \mathrm{H}), 6.95(\mathrm{~d}, J=7.2 \mathrm{~Hz}, 1 \mathrm{H}), 4.83(\mathrm{~d}, J=7.6 \mathrm{~Hz}, 1 \mathrm{H}), 4.58-$ $4.55(\mathrm{~m}, 1 \mathrm{H}), 2.81-2.66(\mathrm{~m}, 2 \mathrm{H}), 1.88-1.71(\mathrm{~m}, 4 \mathrm{H}) ;[\alpha]_{\mathrm{D}}{ }^{25}+34.1^{\circ}\left(c 0.64, \mathrm{CHCl}_{3}, 73 \%\right.$ ee), Lit. $[\alpha]_{\mathrm{D}} 44.3^{\circ}\left(\mathrm{c} 1.40, \mathrm{CHCl}_{3}\right) ;^{2} \mathrm{HPLC}$ analysis: $73 \%$ ee. Chiralcel AD-H, $25.0 \% \mathrm{i}$ PrOH, $0.7 \mathrm{~mL} / \mathrm{min}, 12.9 \mathrm{~min}$ (major), $19.6 \mathrm{~min}$ (minor). The NMR data are consistent with the published data. ${ }^{3}$

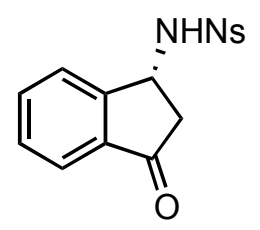

(R)- $N$-(3-Oxo-indan-1-yl)-4-nitrobenzenesulfonamide (7d). yellow solid, $\mathrm{mp}=198$ $200{ }^{\circ} \mathrm{C} ; \mathrm{R}_{\mathrm{f}}=0.40\left(2: 1\right.$ hexanes:EtOAc); $[\alpha]_{\mathrm{D}}^{25}-10.5^{\circ}$ ( $c 0.19$, acetone, $76 \%$ ee); FTIR: 
2966, 2906, 2854, 1707, 1530, 1349, 1259,1166, 1067, 854, $736 \mathrm{~cm}^{-1}$; ${ }^{1} \mathrm{H}$ NMR (DMSO, $500 \mathrm{MHz}) \delta 8.73(\mathrm{~d}, J=8.5 \mathrm{~Hz}, 1 \mathrm{H}), 8.47(\mathrm{~d}, J=9.0 \mathrm{~Hz}, 2 \mathrm{H}), 8.13(\mathrm{~d}, J=9.0 \mathrm{~Hz}, 2 \mathrm{H})$, $7.74(\mathrm{t}, J=7.5 \mathrm{~Hz}, 1 \mathrm{H}), 7.64(\mathrm{~d}, J=8.0 \mathrm{~Hz}, 1 \mathrm{H}), 7.54(\mathrm{~m}, 2 \mathrm{H}), 5.10(\mathrm{~m}, 1 \mathrm{H}), 2.80(\mathrm{dd}, J$ $=19.0,8.0 \mathrm{~Hz}, 1 \mathrm{H}), 2.20(\mathrm{dd}, J=19.0,3.5 \mathrm{~Hz}, 1 \mathrm{H}) ;{ }^{13} \mathrm{C} \mathrm{NMR}\left(125 \mathrm{MHz}, \mathrm{CDCl}_{3}\right) \delta 202.0$ (C), $153.2(\mathrm{C}), 149.7(\mathrm{C}), 136.0(\mathrm{CH}), 135.5(\mathrm{C}), 129.2(\mathrm{CH}), 128.1(\mathrm{CH}), 126.2(\mathrm{CH})$, 124.8 (CH), $122.5(\mathrm{CH}), 50.9(\mathrm{CH}), 43.8(\mathrm{CH})$; Anal. Calcd for $\mathrm{C}_{15} \mathrm{H}_{12} \mathrm{~N}_{2} \mathrm{O}_{5} \mathrm{~S}: \mathrm{C}, 54.21$; H, 3.64; N, 8.43. Found: C, 54.40; H, 3.55; N, 8.39; HPLC analysis: $76 \%$ ee. Chiralcel OJ, 5\% $i$-PrOH, $0.8 \mathrm{~mL} / \mathrm{min}, 5.4 \mathrm{~min}$ (minor), $7.1 \mathrm{~min}$ (major).

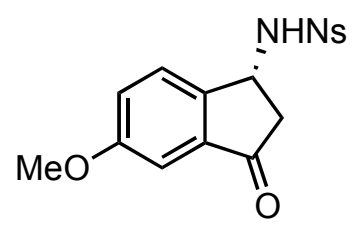

(R)-N-(5-Methoxy-3-oxo-indan-1-yl)-4-nitrobenzenesulfonamide (7e). yellow solid, $m p=165-167^{\circ} \mathrm{C} ; \mathrm{R}_{\mathrm{f}}=0.37(2: 1 \mathrm{Hex}:$ EtOAc $) ;[\alpha]_{\mathrm{D}}{ }^{25}-20.9^{\circ}(c 0.45$, acetone, $74 \%$ ee); FTIR: $3305,1711,1692,1527,1493,1350,1285,1155,1089,855,740,669,616 \mathrm{~cm}^{-1}$; ${ }^{1} \mathrm{H}$ NMR (DMSO, $\left.500 \mathrm{MHz}\right) \delta 8.66(\mathrm{~d}, J=8.5 \mathrm{~Hz}, 1 \mathrm{H}), 8.47(\mathrm{~d}, J=8.5 \mathrm{~Hz}, 2 \mathrm{H}), 8.09$ (d, $J=8.5 \mathrm{~Hz}, 2 \mathrm{H}), 7.37(\mathrm{~d}, J=8.0 \mathrm{~Hz}, 1 \mathrm{H}), 7.28(\mathrm{dd}, J=2.5,8.5 \mathrm{~Hz}, 1 \mathrm{H}), 7.06(\mathrm{~s}, 1 \mathrm{H})$, $5.00(\mathrm{~m}, 1 \mathrm{H}), 3.79(\mathrm{~s}, 3 \mathrm{H}), 2.80(\mathrm{dd}, J=18.5,7.0 \mathrm{~Hz}, 1 \mathrm{H}), 2.20(\mathrm{dd}, J=18.5,3.0 \mathrm{~Hz}$, 1H); ${ }^{13} \mathrm{C}$ NMR (125 MHz, CDCl $)$ o 201.8 (C), 160.3 (C), 149.7 (C), 146.9 (C), 145.7 (C), $137.5(\mathrm{C}), 128.1(\mathrm{CH}), 127.2(\mathrm{CH}), 124.8(\mathrm{CH}), 123.7(\mathrm{CH}), 104.4(\mathrm{CH}), 55.7$ (CH3), $50.5(\mathrm{CH}), 44.4(\mathrm{CH} 2)$; HRMS (ESI) $\mathrm{m} / z$ calc for $\left[\mathrm{C}_{16} \mathrm{H}_{14} \mathrm{~N}_{2} \mathrm{O}_{6} \mathrm{SNa}\right]^{+}(\mathrm{M}+\mathrm{Na})^{+}$ 385.0465. Found: 385.0460; HPLC analysis: $74 \%$ ee: Chiralcel OJ, $5.0 \% i$-PrOH, 0.8 $\mathrm{mL} / \mathrm{min}, 6.9 \min$ (major), $12.4 \mathrm{~min}$ (minor). 


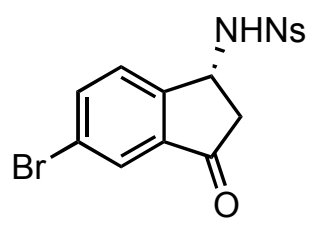

(R)-N-(5-Bromo-3-oxo-indan-1-yl)-4-nitrobenzenesulfonamide (7f). yellow sticky solid; $\mathrm{R}_{\mathrm{f}}=0.47\left(2: 1 \mathrm{Hex}:\right.$ EtOAc); $[\alpha]_{\mathrm{D}}^{25}-6.25^{\circ}$ ( 0.32 , acetone, $73 \%$ ee); FTIR: 3419 , 2360, 2325, 1653, 1023, $762 \mathrm{~cm}^{-1} ;{ }^{1} \mathrm{H}$ NMR (DMSO, $\left.500 \mathrm{MHz}\right) \delta 8.76(\mathrm{bs}, 1 \mathrm{H}), 8.46(\mathrm{~d}$, $J=8.5 \mathrm{~Hz}, 2 \mathrm{H}), 8.11(\mathrm{~d}, J=8.5 \mathrm{~Hz}, 2 \mathrm{H}), 7.90(\mathrm{~d}, J=8.0 \mathrm{~Hz}, 1 \mathrm{H}), 7.78(\mathrm{~s}, 1 \mathrm{H}), 7.45(\mathrm{~d}, J$ $=8.0 \mathrm{~Hz}, 1 \mathrm{H}), 5.06(\mathrm{bs}, 1 \mathrm{H}), 2.83(\mathrm{dd}, J=7.5,18.5 \mathrm{~Hz}, 1 \mathrm{H}), 2.20(\mathrm{dd}, J=3.0,18.5 \mathrm{~Hz}$, $1 \mathrm{H}) ;{ }^{13} \mathrm{C}$ NMR $\left(125 \mathrm{MHz}, \mathrm{CDCl}_{3}\right) \delta 200.6,152.1,149.7,146.6,138.0,137.8,128.4$, 128.1, 125.0, 124.7, 122.7, 50.7, 43.9; HRMS (EI) $m / z$ calc for $\left[\mathrm{C}_{15} \mathrm{H}_{11} \mathrm{BrN}_{2} \mathrm{O}_{5} \mathrm{~S}\right]^{+}\left(\mathrm{M}^{+}\right)$: 409.9567. Found: 409.9576; HPLC analysis: $73 \%$ ee. Chiralcel OJ, $5.0 \% i$-PrOH, 0.8 $\mathrm{mL} / \mathrm{min}, 5.4 \mathrm{~min}$ (major), $7.1 \mathrm{~min}$ (minor).<smiles>NN[C@H]1CCC(=O)c2ccccc21</smiles>

(R)-N-(4-Oxo-1,2,3,4-tetrahydronaphthalene-1-yl)-4-nitrobenzenesulfonamide (7g). $\mathrm{R}_{\mathrm{f}}=0.40$ (2:1 hexanes:EtOAc); $[\alpha]_{\mathrm{D}}^{25}-13.4^{\circ}$ (c 1.02, acetone, $78 \%$ ee); FTIR: 2966, 2906, 2854, 1707, 1530, 1349, 1259,1166, 1067, 854, $736 \mathrm{~cm}^{-1} ;{ }^{1} \mathrm{H}$ NMR (DMSO, 300 MHz) $\delta 8.75(\mathrm{~d}, \mathrm{~J}=8.7 \mathrm{~Hz}, 1 \mathrm{H}), 8.44(\mathrm{~d}, J=8.7 \mathrm{~Hz}, 2 \mathrm{H}), 8.14(\mathrm{~d}, J=8.7 \mathrm{~Hz}, 2 \mathrm{H}), 7.84$ (d, $J=7.8 \mathrm{~Hz}, 1 \mathrm{H}), 7.61(\mathrm{t}, J=7.8 \mathrm{~Hz}, 1 \mathrm{H}), 7.43(\mathrm{t}, J=7.5 \mathrm{~Hz}, 1 \mathrm{H}), 7.34(\mathrm{~d}, J=7.8 \mathrm{~Hz}$, 1H), $4.78(\mathrm{~m}, 1 \mathrm{H}), 2.61(\mathrm{~m}, 2 \mathrm{H}), 1.93(\mathrm{~m}, 2 \mathrm{H}) ;{ }^{13} \mathrm{C}$ NMR (125 MHz, DMSO) $\delta$ 196.1, 149.6, 147.4, 143.1, 133.8, 131.5, 128.1, 127.9, 127.7, 126.3, 124.7, 51.2, 35.2, 29.5; HRMS (EI) $m / z$ calc for $\left[\mathrm{C}_{16} \mathrm{H}_{14} \mathrm{~N}_{2} \mathrm{O}_{5} \mathrm{~S}\right]^{+}\left(\mathrm{M}^{+}\right)$: 346.0623. Found: 346.0634; HPLC 
analysis: $78 \%$ ee. Chiralcel OJ, $5.0 \%$ i-Propanol, $0.8 \mathrm{~mL} / \mathrm{min}, 15.4 \mathrm{~min}$ (minor), 18.1 $\min$ (major).

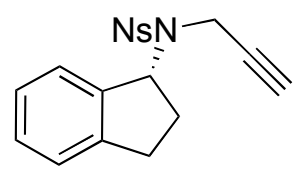

(R)- $N$-Indan-1-yl-4-nitro- $N$-prop-2-ynyl-benzenesulfonamide (8). light yellow solid, $\mathrm{mp}=102-105^{\circ} \mathrm{C} ; \mathrm{R}_{\mathrm{f}}=0.40(5: 1$ hexanes:EtOAc $) ;[\alpha]_{\mathrm{D}}^{25}-12.3^{\circ}\left(c 1.17, \mathrm{CHCl}_{3}\right) ; \mathrm{FTIR}:$ $3281,3101,2946,1528,1348,1158,1093,855,737,684 \mathrm{~cm}^{-1} ;{ }^{1} \mathrm{H}$ NMR $\left(\mathrm{CDCl}_{3}, 500\right.$ MHz) $\delta 8.40(\mathrm{~d}, J=7.0 \mathrm{~Hz}, 2 \mathrm{H}), 8.22(\mathrm{~d}, J=7.0 \mathrm{~Hz}, 2 \mathrm{H}), 7.29-7.15(\mathrm{~m}, 4 \mathrm{H}), 5.61$ (t, $J=$ $7.5 \mathrm{~Hz}, 1 \mathrm{H}), 4.28(\mathrm{dd}, J=19.0,2.5 \mathrm{~Hz}, 1 \mathrm{H}), 3.60(\mathrm{dd}, J=19.0,2.5 \mathrm{~Hz}, 1 \mathrm{H}), 3.04(\mathrm{~m}$, 1H), $2.83(\mathrm{~m}, 1 \mathrm{H}), 2.23(\mathrm{~m}, 2 \mathrm{H}), 2.12(\mathrm{~s}, 1 \mathrm{H}) ;{ }^{13} \mathrm{C} \mathrm{NMR}\left(125 \mathrm{MHz}, \mathrm{CDCl}_{3}\right) \delta 149.9(\mathrm{C})$, $146.5(\mathrm{C}), 143.6(\mathrm{C}), 138.8(\mathrm{C}), 128.9(\mathrm{CH}), 128.8(\mathrm{CH}), 127.1(\mathrm{CH}), 125.1(\mathrm{CH}), 124.5$ $(\mathrm{CH}), 124.1(\mathrm{CH}), 78.9(\mathrm{C}), 72.9(\mathrm{C}), 63.8(\mathrm{CH}), 32.8(\mathrm{CH} 2), 30.1(\mathrm{CH} 2), 29.1(\mathrm{CH} 2)$; HRMS (EI) $m / z$ calc for $\left[\mathrm{C}_{18} \mathrm{H}_{16} \mathrm{~N}_{2} \mathrm{O}_{4} \mathrm{~S}\right]^{+}\left(\mathrm{M}^{+}\right)$: 356.0825. Found: 356.0819 .

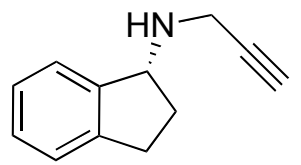

(R)-Indan-1-yl-prop-2-ynyl-amine (9). solid, $\quad \mathrm{mp}=148 \quad{ }^{\circ} \mathrm{C} \quad \mathrm{R}_{\mathrm{f}}=0.33 \quad(3: 1$ hexanes:EtOAc); $[\alpha]_{\mathrm{D}}{ }^{25}+18.8^{\circ}\left(c\right.$ 1.7, $\left.\mathrm{CHCl}_{3}\right)$; FTIR: 3281, 2929, 2848, 1456, 1349, 1161, 1088, $649 \mathrm{~cm}^{-1} ;{ }^{1} \mathrm{H}$ NMR $\left(\mathrm{CDCl}_{3}, 300 \mathrm{MHz}\right) \delta$ 7.57-7.54(m, $\left.1 \mathrm{H}\right)$, 7.46-7.39 (m, $3 \mathrm{H}), 4.62(\mathrm{t}, \mathrm{J}=10 \mathrm{~Hz}, 1 \mathrm{H}), 3.73(\mathrm{~s}, 2 \mathrm{H}), 3.25(\mathrm{~m}, 1 \mathrm{H}), 3.06(\mathrm{~m}, 1 \mathrm{H}), 2.62(\mathrm{~m}, 1 \mathrm{H}), 2.46$ (s, 1H), $2.12(\mathrm{~m}, 2 \mathrm{H}) ;{ }^{13} \mathrm{C}$ NMR $\left(125 \mathrm{MHz}, \mathrm{CDCl}_{3}\right) \delta 144.5(\mathrm{C}), 143.8(\mathrm{C}), 127.6(\mathrm{CH})$, $126.2(\mathrm{CH}), 124.8(\mathrm{CH}), 124.2(\mathrm{CH}), 82.5(\mathrm{C}), 71.3(\mathrm{C}), 61.9(\mathrm{CH}), 36.1(\mathrm{CH} 2), 33.3$ (CH2), $30.4(\mathrm{CH} 2)$; HRMS (ESI) $m / z$ calc for $\left[\mathrm{C}_{12} \mathrm{H}_{13} \mathrm{NNa}\right]^{+}\left([\mathrm{M}+\mathrm{Na}]^{+}\right)$: 194.0946 . Found: 194.0932. 


\section{General Procedure for Intramolecular C-H Amination:}

To a solution of $N$-tosyloxycarbamate $(0.5 \mathrm{mmol})$ in dichloromethane $(10.0 \mathrm{~mL})$, were added $\mathrm{K}_{2} \mathrm{CO}_{3}$ (1.5 mmol, 3 equiv.) and $\mathrm{Rh}_{2}(S \text {-TCPTAD })_{4}(0.01 \mathrm{mmol})$. The resulting suspension was stirred at $23{ }^{\circ} \mathrm{C}$ for $4 \mathrm{~h}$. The mixture was filtered to remove the precipitate and the solvent was removed under vacuum. The crude reaction mixture was then purified by flash chromatography.

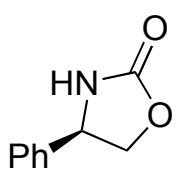

(R)-4-Phenyloxazolidin-2-one (11a). ${ }^{1} \mathrm{H}$ NMR (400 MHz, $\left.\mathrm{CDCl}_{3}\right) \delta$ 7.42-7.32 (m, 5H), $6.11(\mathrm{bs}, 1 \mathrm{H}), 4.95(\mathrm{t}, J=7.6 \mathrm{~Hz}, 1 \mathrm{H}), 4.72(\mathrm{t}, J=8.8 \mathrm{~Hz}, 1 \mathrm{H}), 4.17(\mathrm{dd}, J=8.8,7.6 \mathrm{~Hz}$, $1 \mathrm{H}) ;[\alpha]_{\mathrm{D}}^{25}-40.8^{\circ}\left(c 0.86, \mathrm{CHCl}_{3}, 82 \%\right.$ ee $)$ Lit. $[\alpha]_{\mathrm{D}}$ for $(R)-4$-phenyloxazolidin-2-one: ${ }^{4}$ $-57.7^{\circ}\left(\mathrm{c} 1.00, \mathrm{CHCl}_{3}\right) ; \quad \mathrm{HPLC}$ analysis. $82 \%$ ee. Chiralcel OD-H, $7 \% i$-PrOH, 0.9 $\mathrm{mL} / \mathrm{min}, 14.0 \mathrm{~min}$ (major), $17.2 \mathrm{~min}$ (minor). The NMR data are consistent with the published data. ${ }^{5}$

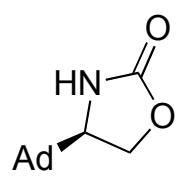

(R)-4-Adamantyloxazolidin-2-one (11b). ${ }^{1} \mathrm{H}$ NMR (500 MHz, $\left.\mathrm{CDCl}_{3}\right) \delta 5.62$ (bs, 1H), $4.30(\mathrm{~d}, J=7.5 \mathrm{~Hz}, 2 \mathrm{H}), 3.40(\mathrm{t}, J=7.2 \mathrm{~Hz}, 1 \mathrm{H}), 2.03(\mathrm{~s}, 3 \mathrm{H}), 1.77-1.51(\mathrm{~m}, 12 \mathrm{H}) ;[\alpha]_{\mathrm{D}}{ }^{25}$ -12.5 (c $0.62, \mathrm{CHCl}_{3}, 78 \%$ ee); lit. $[\alpha]_{\mathrm{D}}$ for $(S)$-4-adamantyloxazolidin-2-one ${ }^{6}:+8.1$ (c 0.78, $\mathrm{CHCl}_{3}$ ); HPLC analysis. $78 \%$ ee. Chiralcel OD-H, $0.9 \mathrm{~mL} / \mathrm{min}, 7 \% i$-PrOH, 13.1 min (minor), 24.7 min (major). The NMR data are consistent with the published data. ${ }^{5}$

\footnotetext{
${ }^{4}$ Evans, D. A.; Sjogren, E. B. Tetrahedron Lett. 1985, 26, 3783.

5 (a) Espino, C.G.; Du Bois, J. Angew. Chem. Int. Ed. 2001, 40, 598. (b) Lebel, H.; Huard, K.; Lectard, S. J. Am. Chem. Soc. 2005, 127, 14198.

${ }^{6}$ Takacs, J. M.; Jaber, M. R.; Vellekoop, A. S. J. Org. Chem. 1998, 63, 2742.
} 


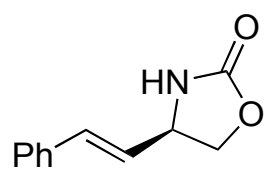

(R)-4-Styryloxazolidin-2-one (11c). ${ }^{1} \mathrm{H}$ NMR $\left(500 \mathrm{MHz}, \mathrm{CDCl}_{3}\right) \delta 7.39-7.30(\mathrm{~m}, 5 \mathrm{H})$, $6.62(\mathrm{~d}, J=16.0 \mathrm{~Hz}, 1 \mathrm{H}), 6.14(\mathrm{dd}, J=16.0,8.0 \mathrm{~Hz}, 1 \mathrm{H}), 5.09$ (bs, 1H), 4.63- 4.54 (m, $2 \mathrm{H}), 4.16(\mathrm{~m}, 1 \mathrm{H}) ;[\alpha]_{\mathrm{D}}^{25}+22.5^{\circ}\left(c 0.26, \mathrm{CHCl}_{3}\right)$, Lit. $[\alpha]_{\mathrm{D}}$ for $(R)$-4-styryloxazolidin-2one: ${ }^{7}+19.3\left(c\right.$ 1.945, $\left.\mathrm{CHCl}_{3}\right)$; HPLC analysis. $79 \%$ ee. Chiralcel OD-H, $10.0 \% i-\mathrm{PrOH}$, $0.9 \mathrm{~mL} / \mathrm{min}, 8.5 \mathrm{~min}$ (minor), $15.3 \mathrm{~min}$ (major). The NMR data are consistent with the published data. ${ }^{5}$

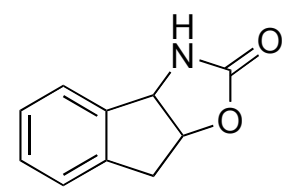

(4R,5S)-Indano[1,2-d]oxazolidin-2-one (11d). ${ }^{1} \mathrm{H}$ NMR $\left(500 \mathrm{MHz}, \mathrm{CDCl}_{3}\right) \delta$ 7.33-7.26 (m, 4H), $6.87(\mathrm{bs}, 1 \mathrm{H}), 5.43-5.40(\mathrm{~m}, 1 \mathrm{H}), 5.17(\mathrm{~d}, J=7.0,1 \mathrm{H}), 3.44-3.34(\mathrm{~m}, 2 \mathrm{H})$; $[\alpha]_{\mathrm{D}}{ }^{25}+12.1^{\circ}\left(c 0.36, \mathrm{CHCl}_{3}, 43 \%\right.$ ee $)$; Lit. $[\alpha]_{\mathrm{D}}$ for $(4 R, 5 S)$-indano[1,2- $\left.d\right]$ oxazolidin-2one: ${ }^{8}+76.9\left(c\right.$ 1.2, $\left.\mathrm{CHCl}_{3}\right)$; HPLC analysis. $43 \%$ ee. Chiralcel AD-H, $7 \% i$-PrOH, 0.9 $\mathrm{mL} / \mathrm{min}$, 17.0 min (major), 29.0 min (minor). The NMR data are consistent with the published data. ${ }^{5}$

\footnotetext{
${ }^{7}$ Sibi, M.P.; Rutherford, D.; Renhowe, P. A.; Li, B. J. Am. Chem. Soc. 1999, 121, 7509.

${ }^{8}$ Ghosh, A. K.; Kincaid, J. F.; Haske, M. G. Synthesis, 1997, 5, 541.
} 


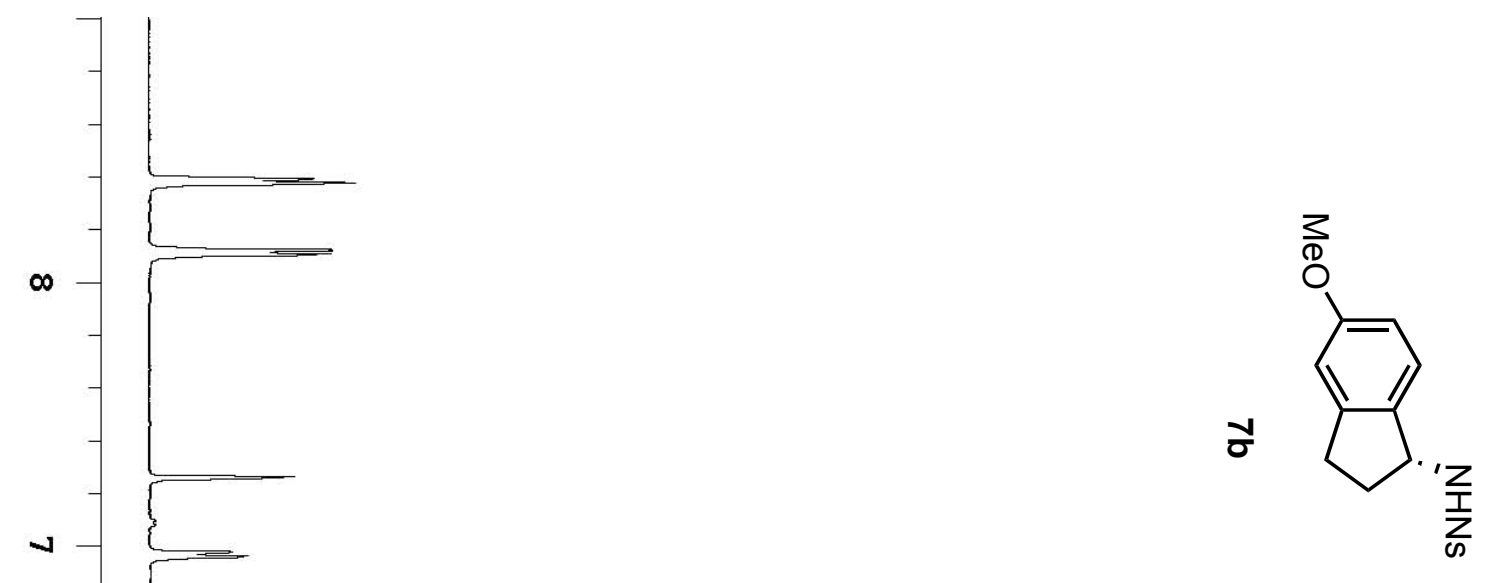



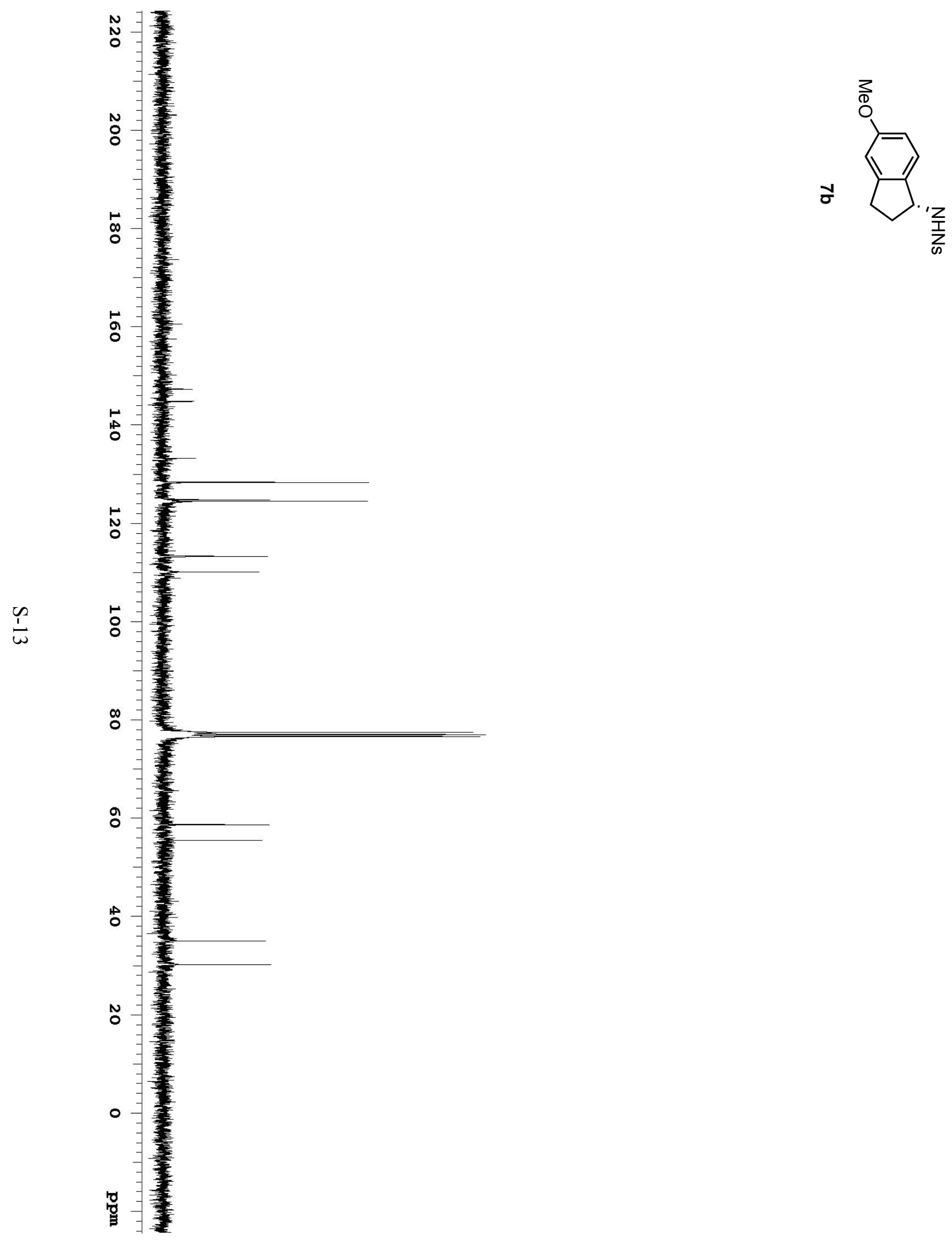


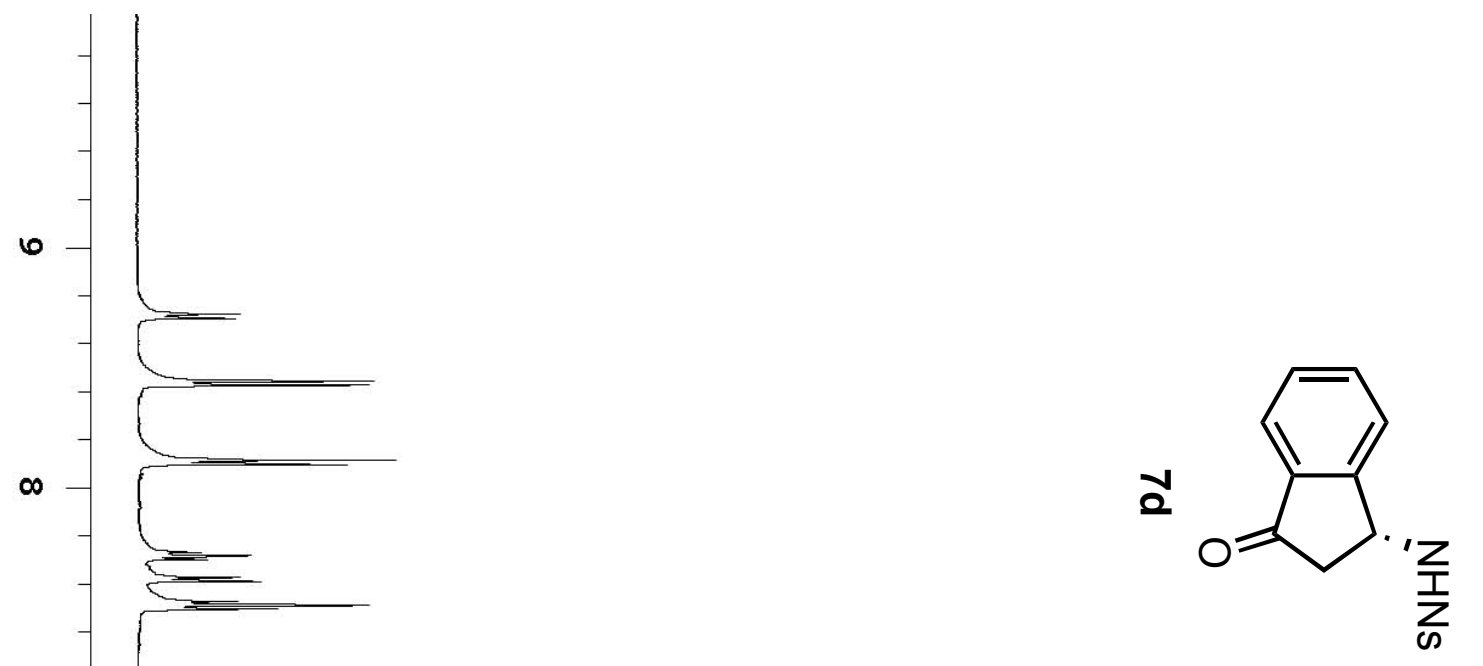

a

$\checkmark$
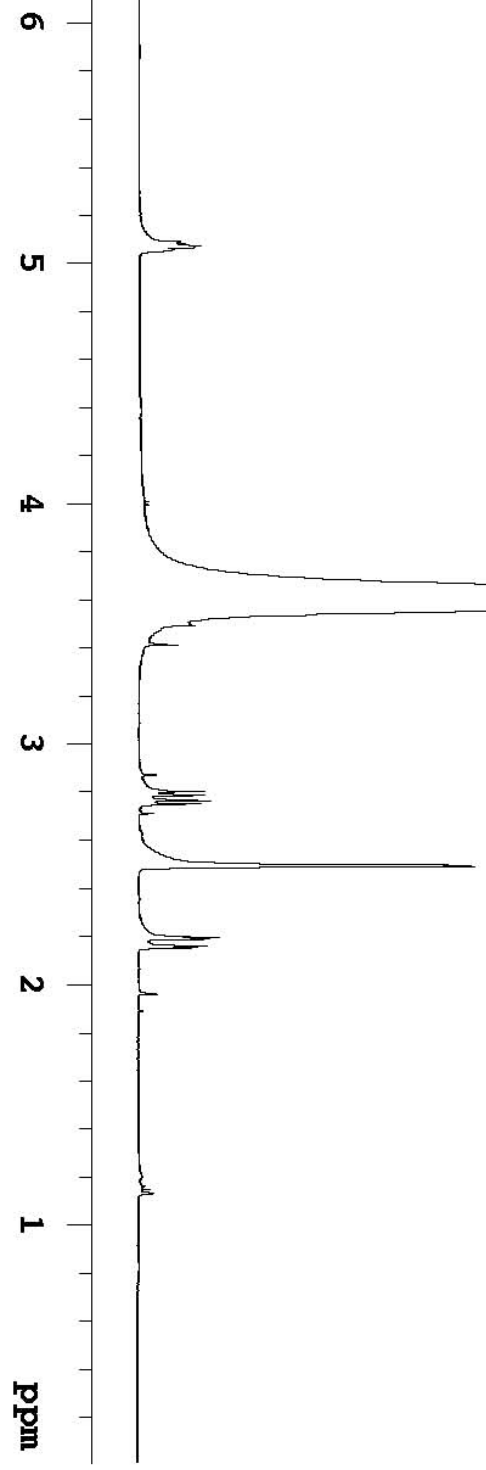


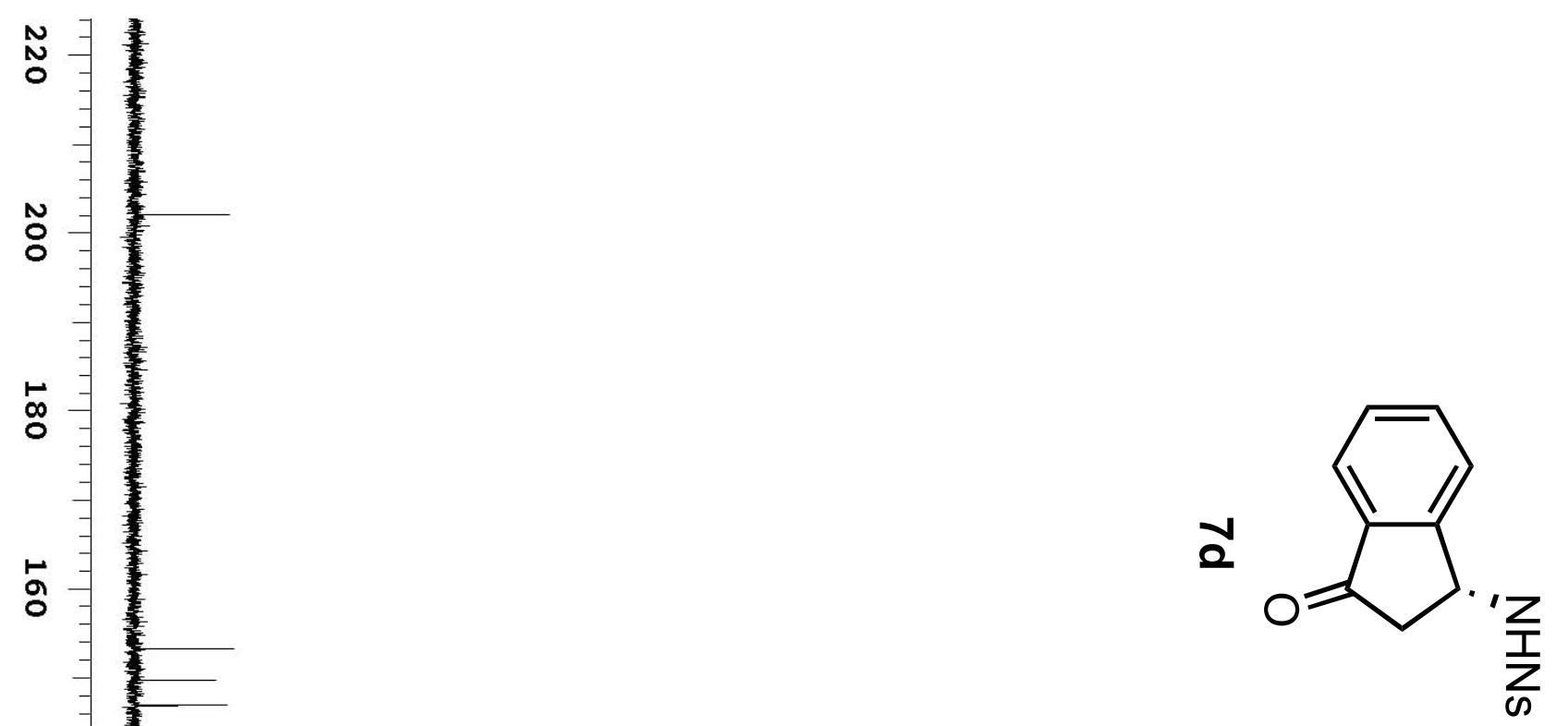




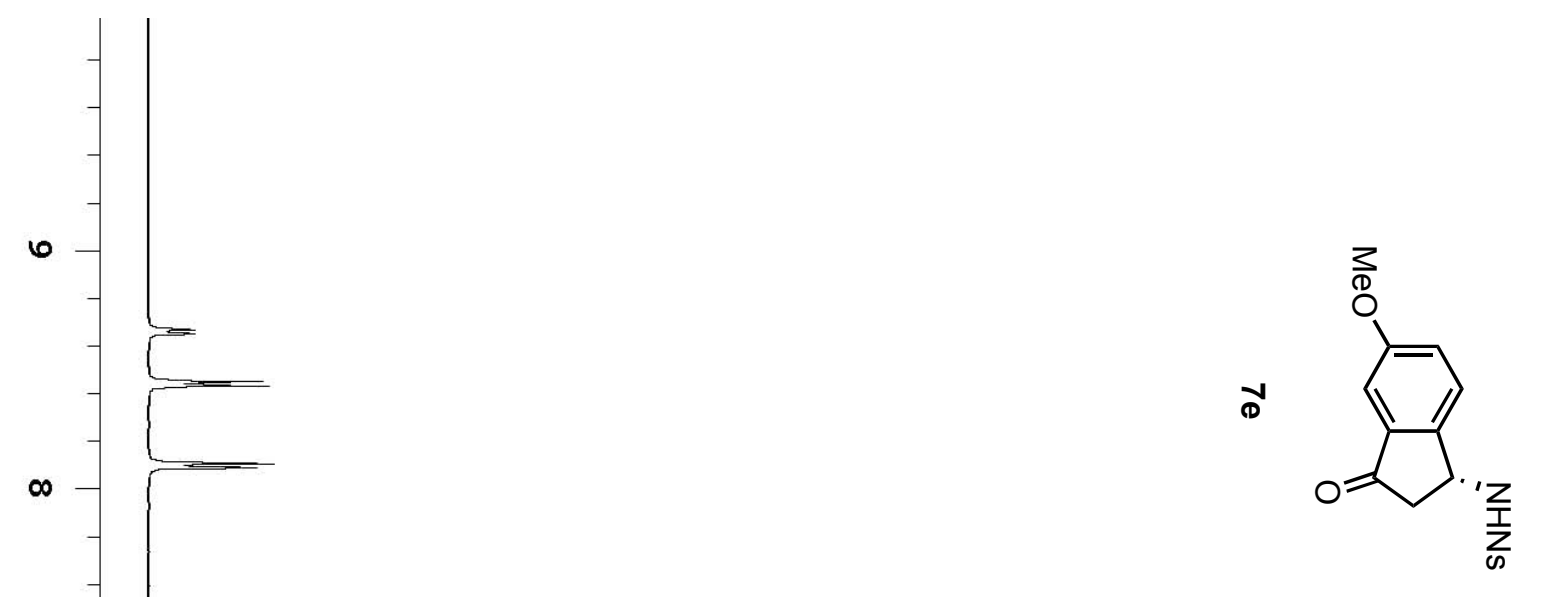




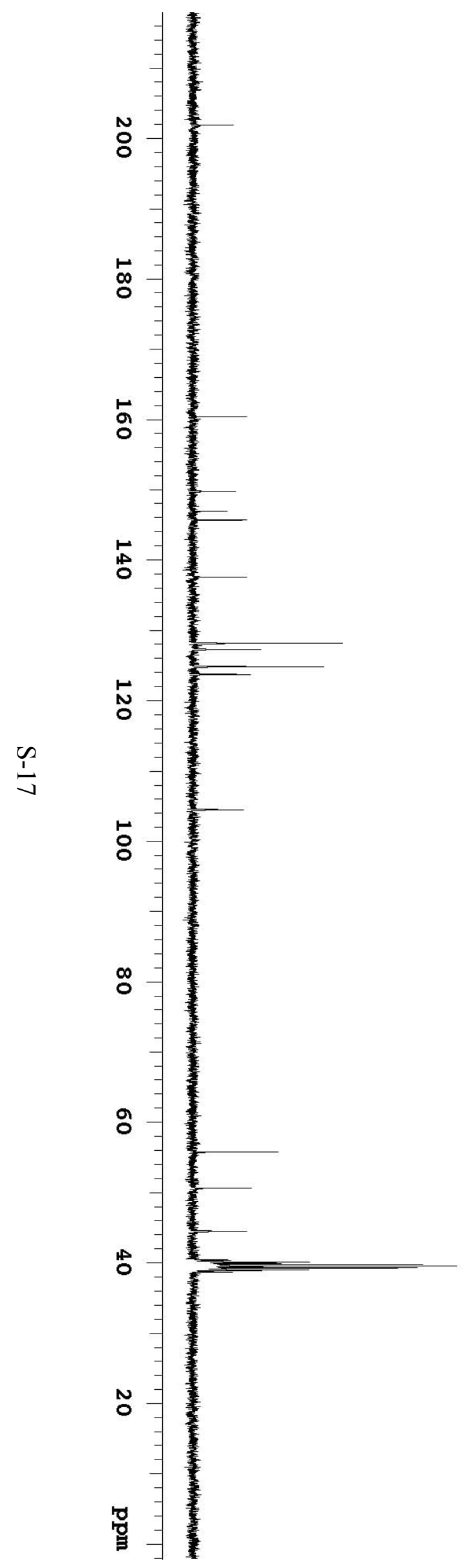




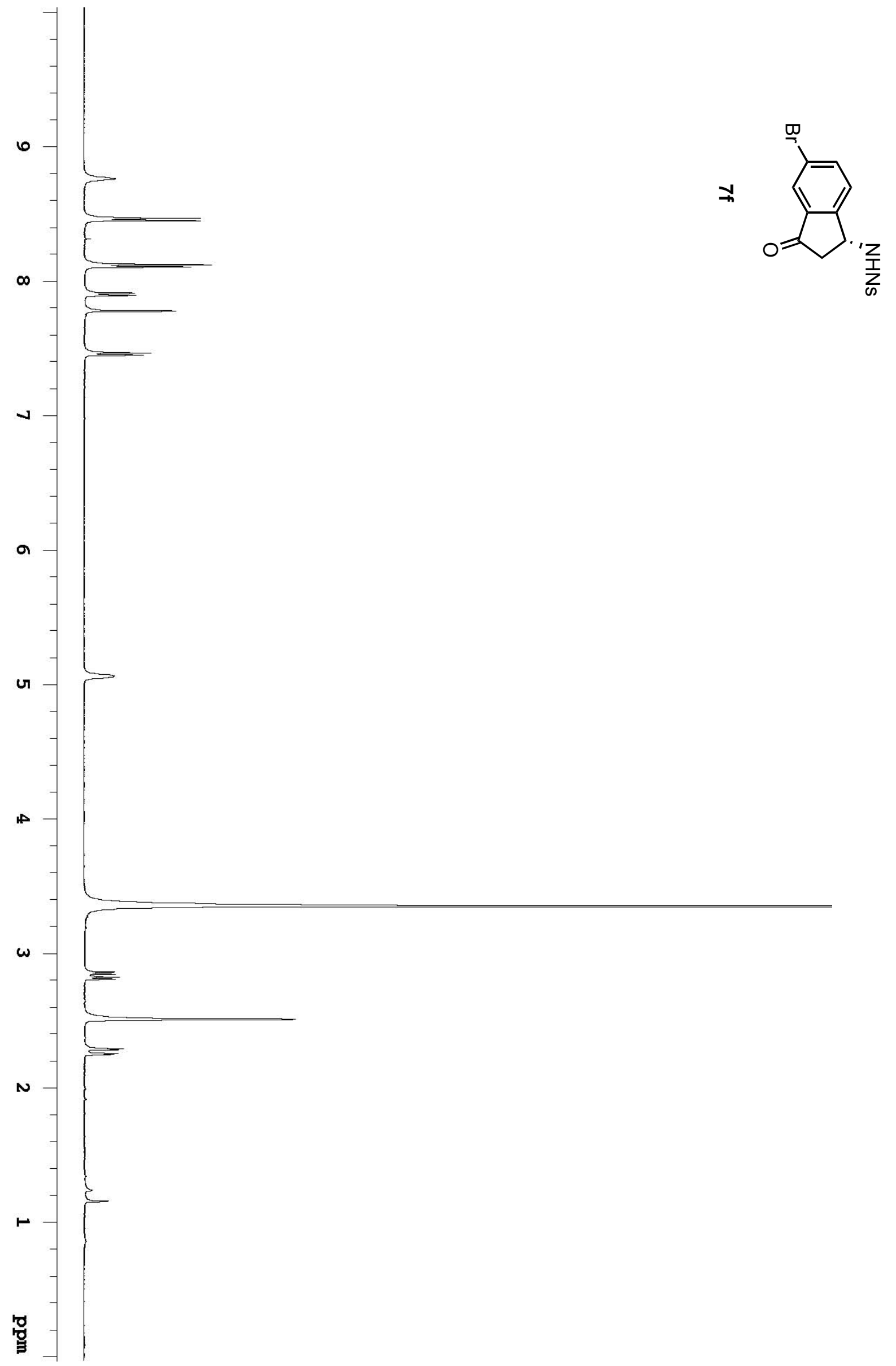




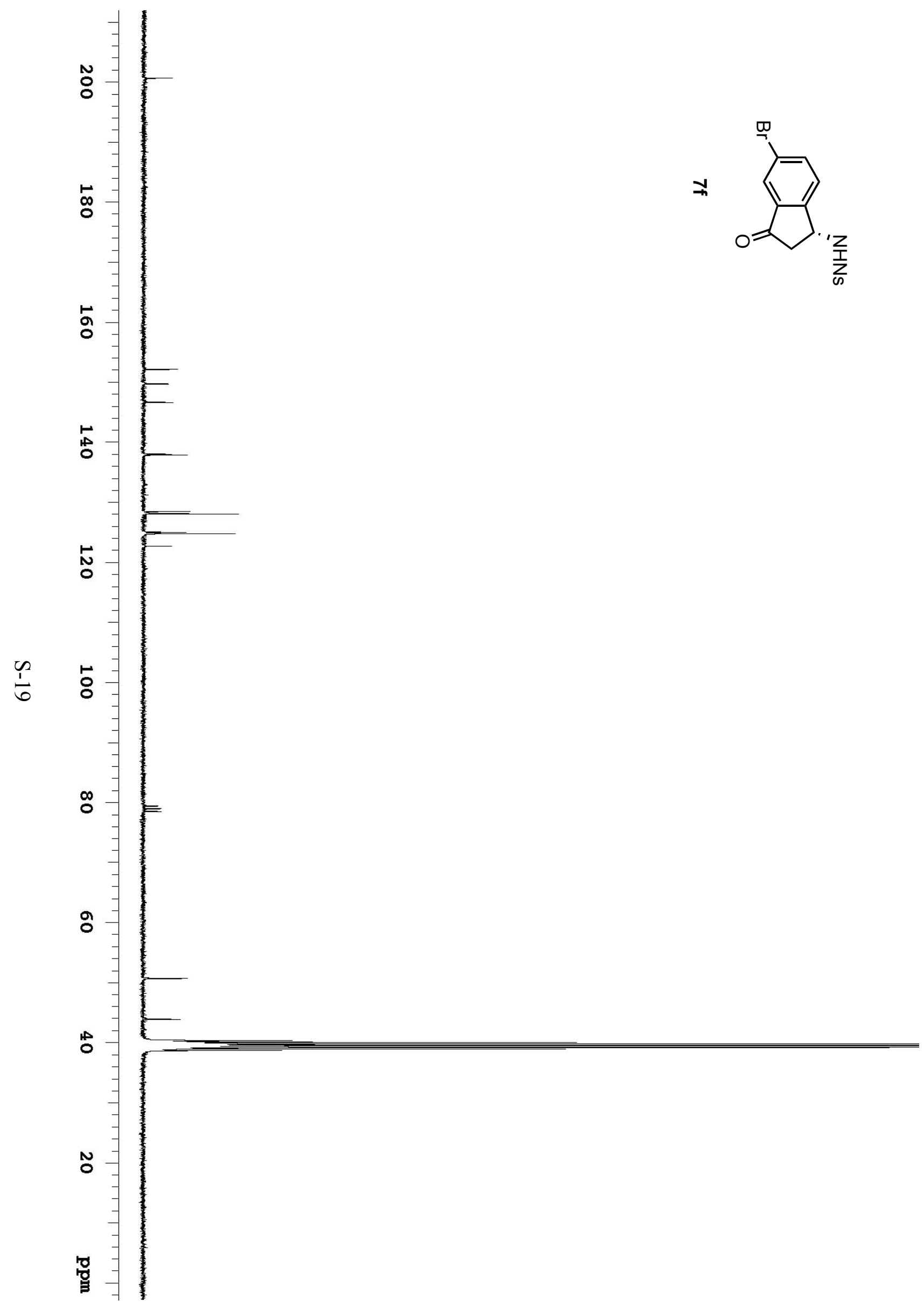




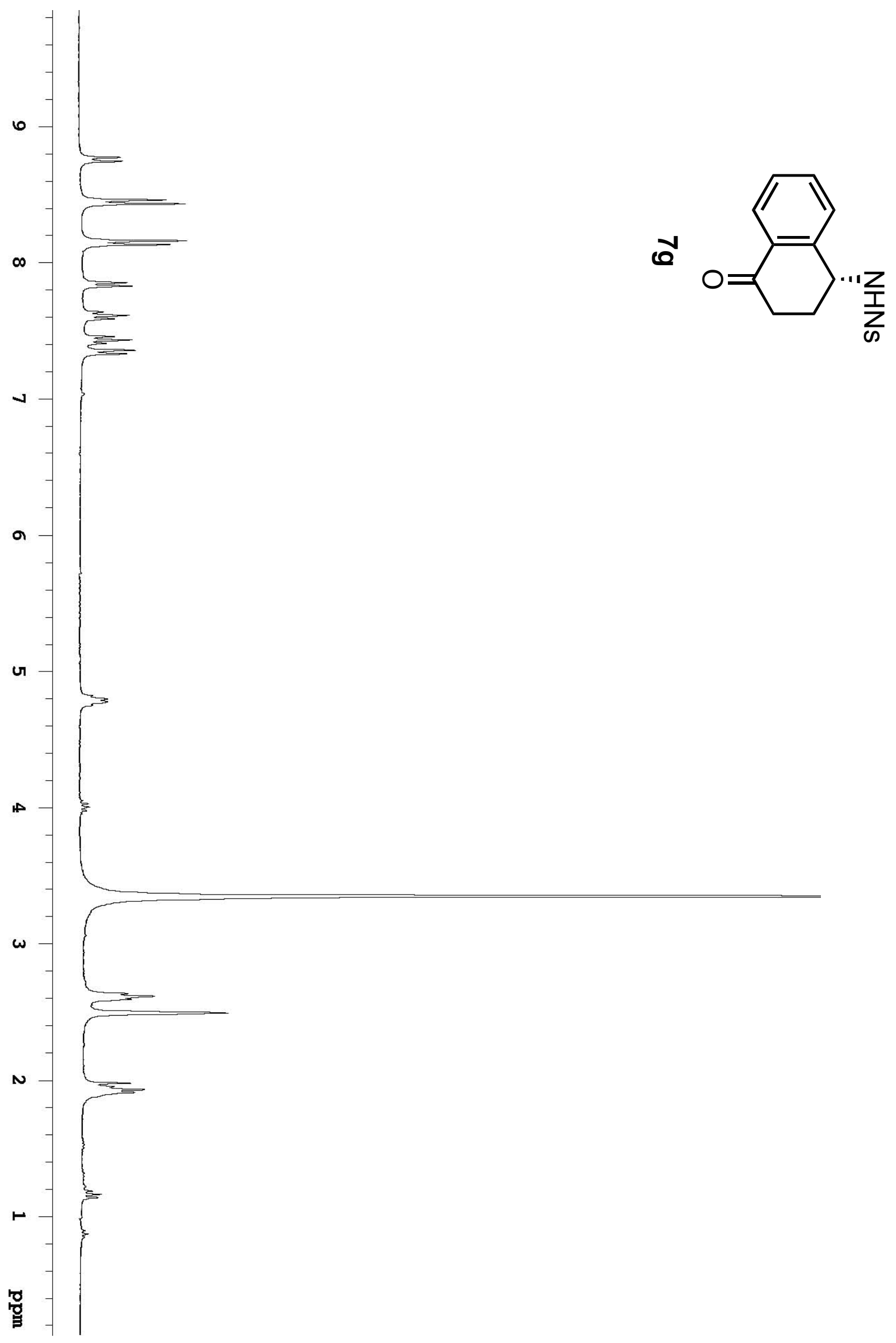




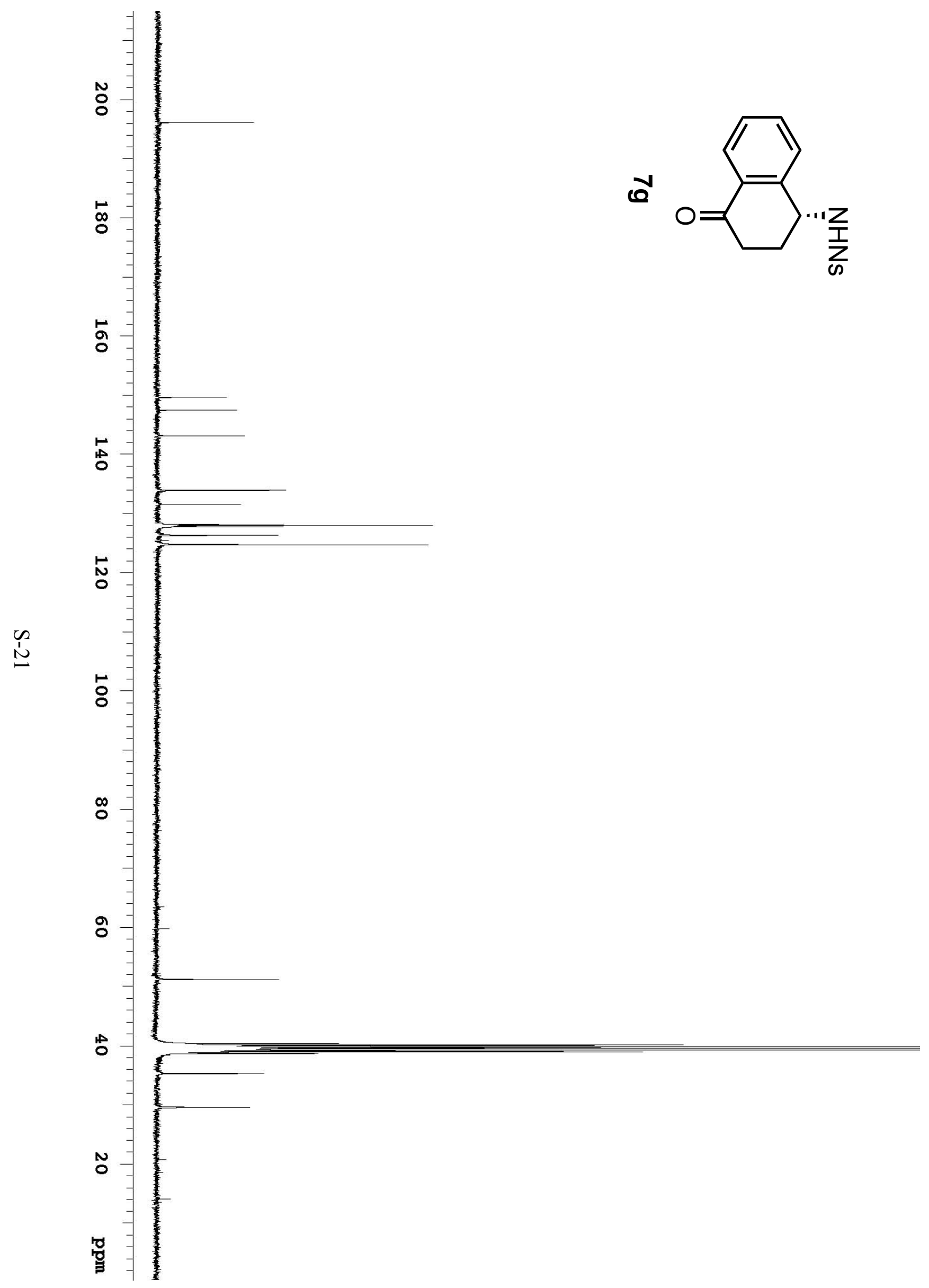



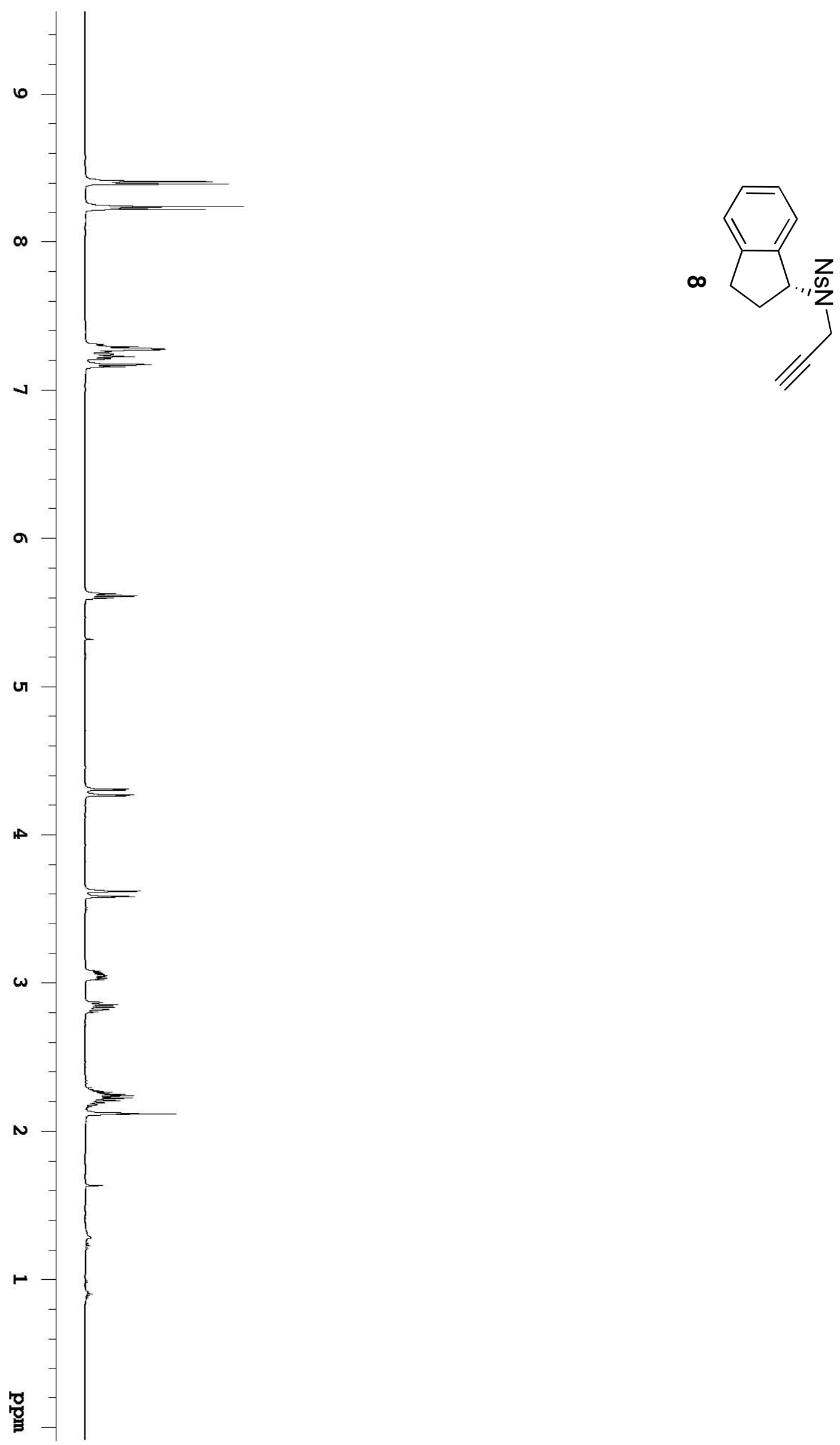


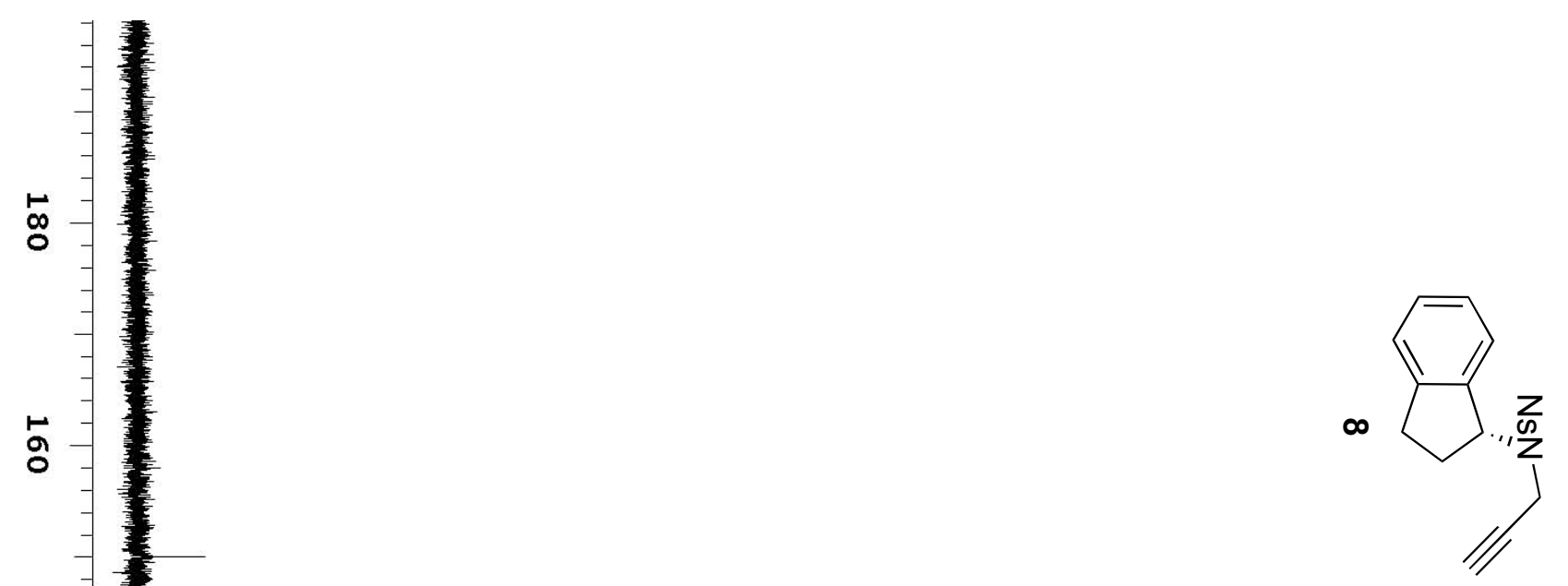



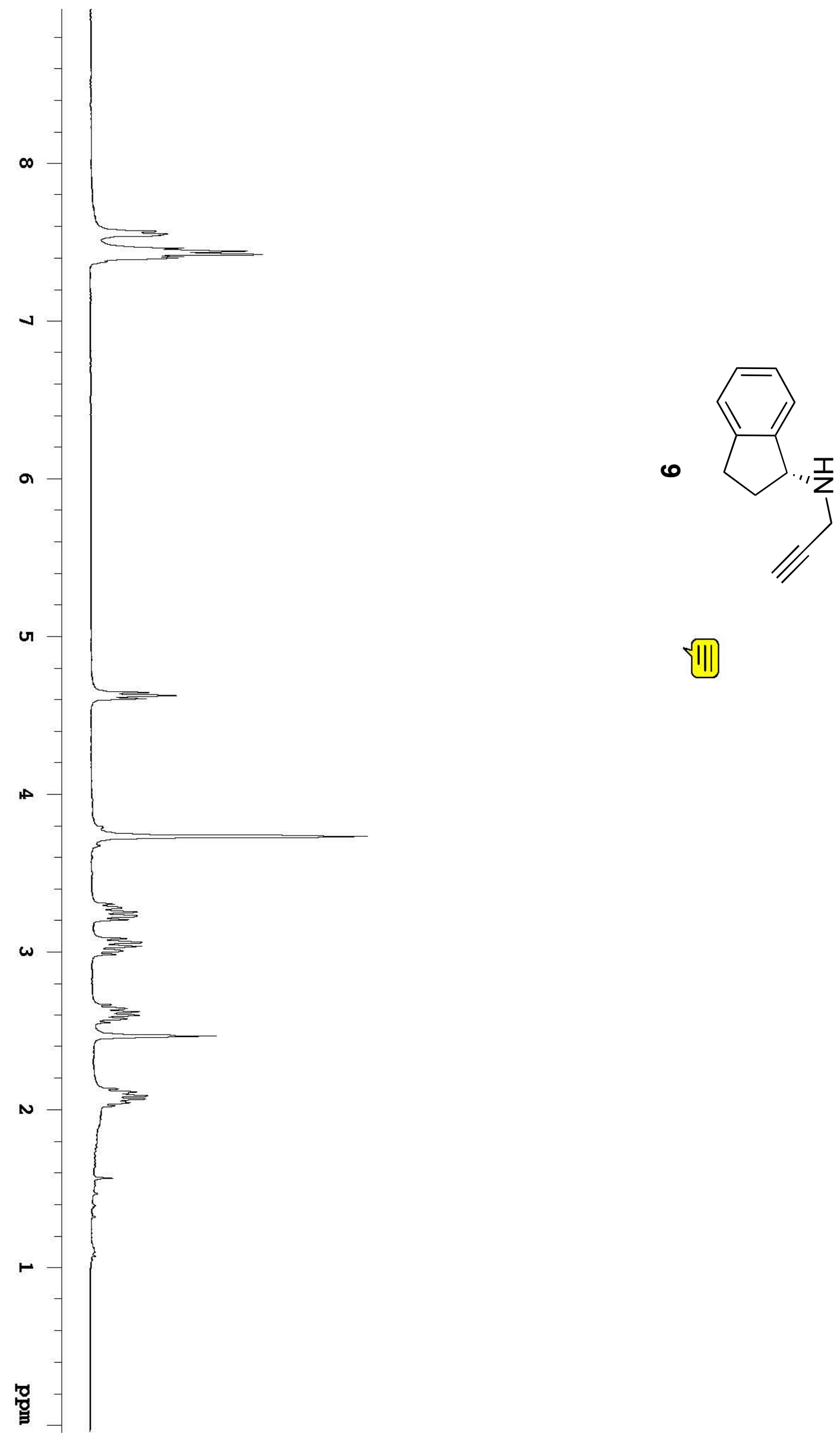


$$
\mid
$$

